\title{
Eine breitangelegte Begleitforschung ist unabdingbar
}

\section{Pierre-François Cuénoud}

Dr. med., Mitglied des Zentralvorstands, Leiter des Ressorts SwissDRG
1 Martin J. DRG: Nutzen und Risiken. Schweiz Ärztezeitung. 2008; 89(28/29):1272.

\section{Lieber Kollege}

Die Fragen, die Sie in Ihrem Beitrag aufwerfen, sind durchaus berechtigt. Über die meisten Ihrer Überlegungen wurde innerhalb der Arbeitsgruppe SwissDRG der FMH bereits ausgiebig diskutiert. Die Ergebnisse dieser Debatte kamen schliesslich im Positionspapier der FMH zum Ausdruck, das vom Zentralvorstand und anschliessend im Februar 2007 von der Delegiertenversammlung gutgeheissen wurde (verfügbar unter www.fmh.ch $\rightarrow$ Unsere Dienstleistungen $\rightarrow$ Tarife $\rightarrow$ SwissDRG).

In diesem Zusammenhang ist zu berücksichtigen, dass das Prinzip der Vergütung von Spitalleistungen auf der Grundlage von Fallpauschalen im Gesetz verankert ist und dass sich im Bereich der Sozialversicherung niemand diesem Prinzip entziehen kann. Es ist im revidierten KVG integriert und dementsprechend auch in der laufenden Überarbeitung des UVG vorgesehen. In der gegenwärtig geplanten Form wird SwissDRG nur für stationäre Behandlungen akuter somatischer Erkrankungen zur Anwendung gelangen. Die Palliativpflege und die Psychiatrie sind derzeit von diesem System ausdrücklich ausgenommen. Trotz bestimmter möglicher Ansätze der Zahlstellen scheint es eher unwahrscheinlich zu sein, dass diese Einschränkung im kurzfristigen Zeitraum in Frage gestellt wird. Denn schon die Entwicklung einer Tarifstruktur, mit der die Realität im Bereich der akuten somatischen Erkrankungen bestmöglich zum Ausdruck kommt, ist eine sehr umfangreiche Aufgabe.

Sie weisen auf das Risiko hin, dass gewisse Akteure versuchen, ihre Interessen bestmöglich zu befriedigen, indem sie die Merkmale des Systems ausnutzen. In allen Ländern, die das DRG-System eingeführt haben, wurde das hinter- listige Phänomen der Überkodierung festgestellt, das für die Patienten im Gegensatz zu den vorzeitigen Entlassungen weniger nachteilige Auswirkungen hat. Doch mit der Zeit nimmt dieses Phänomen $\mathrm{ab}$ und stabilisiert sich auf einem «vertretbaren» Niveau. Dies ist nicht zuletzt dem Umstand zu verdanken, dass mit der Kodierung Spezialisten beauftragt werden, die unabhängig für mehrere Spitäler tätig sind. Da es um beträchtliche Summen geht, versuchen die Zahlstellen, eine systematische Kontrolle dieser Kodierung auf mehreren Ebenen durchzusetzen, während sich die Leistungserbringer für eine einzige Instanz aussprechen, die alle Akteure umfasst und die Kontrolltätigkeit mit Hilfe von Stichproben ausübt.

Grundsätzlich steht ausser Zweifel, dass die Einführung der neuen SwissDRG-Tarifstruktur zahlreiche Änderungen zur Folge haben wird: beim Verhalten der Leistungserbringer und $\mathrm{Pa}$ tienten, in der schweizerischen Spitallandschaft, bei den Beziehungen mit der ambulanten Versorgung und in bezug auf den Einfluss der ökonomischen Logik. Damit die Beibehaltung der Qualität in der gesamten Therapiekette gewährleistet werden kann, muss unbedingt eine breitangelegte Begleitforschung aller resultierenden Änderungen vorgesehen werden (Änderungen auf menschlicher, sozialer, ethischer, geographischer, beruflicher, wirtschaftlicher Ebene usw.). Dazu muss eine Bestandesaufnahme vor und nach der Einführung des neuen Tarifs vorgenommen werden. Ein derartiges Projekt ist ein ambitiöses Vorhaben, das umfangreiche Mittel erfordert. Aus diesem Grund hat die FMH vorgeschlagen, dass es mit einem Fallpauschalenzuschlag finanziert und partnerschaftlich mit der SwissDRG AG geführt wird. 